Overdetermined problems for $\mathrm{p}$-Laplace and generalized Monge-Ampére equations

\author{
Emamizadeh, B., Liu, Y., Porru, G.
}

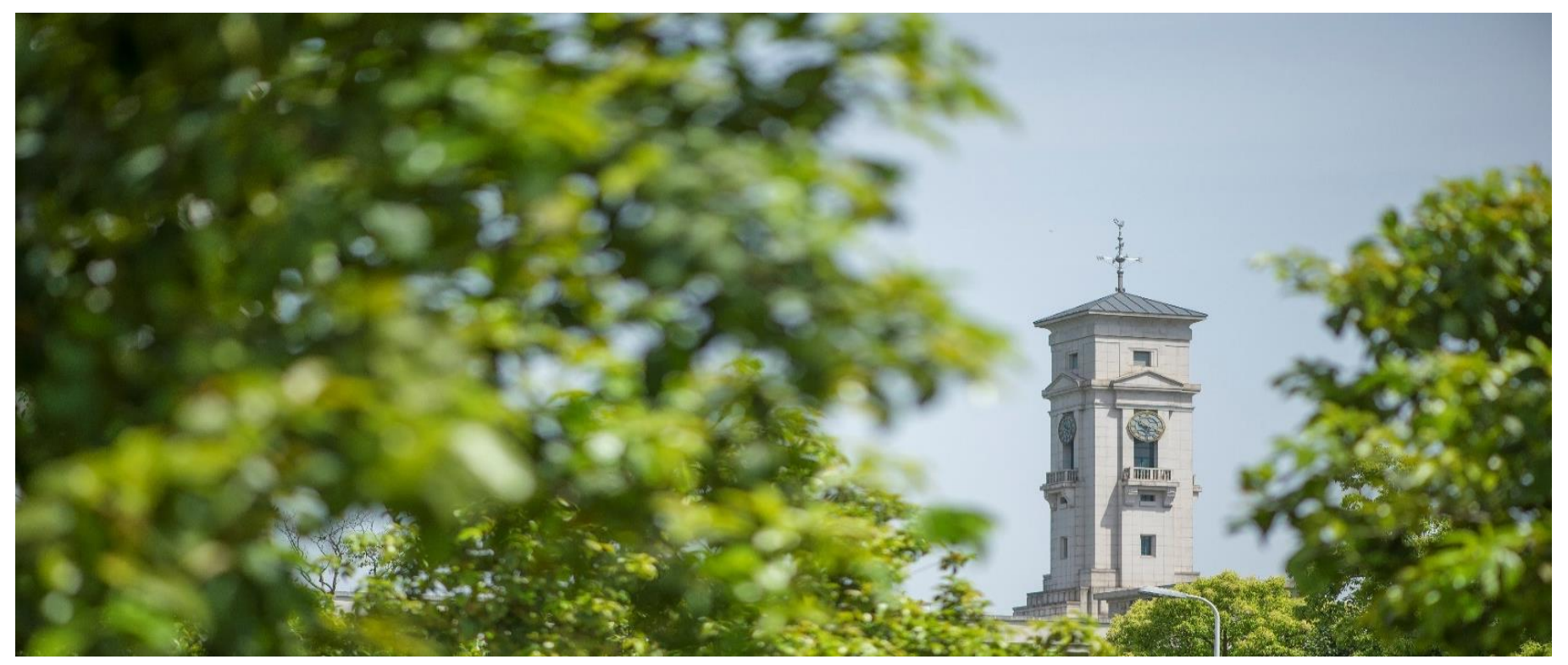


University of Nottingham Ningbo China, 199 Taikang East Road, Ningbo, 315100, China

First published 2020

This work is made available under the terms of the Creative Commons Attribution 4.0 International License:

http://creativecommons.org/licenses/by/4.0

The work is licenced to the University of Nottingham Ningbo China under the Global University Publication Licence:

https://www.nottingham.edu.cn/en/library/documents/researchsupport/global-university-publications-licence.pdf Nottingham 


\title{
Overdetermined problems for p-Laplace and generalized Monge-Ampére equations
}

\author{
Behrouz Emamizadeh, Yichen Liu †, Giovanni Porru ${ }^{\ddagger}$
}

\begin{abstract}
We investigate overdetermined problems for p-Laplace and generalized Monge-Ampére equations. By using the theory of domain derivative we find duality results and a characterization of the overdetermined boundary conditions via minimization of suitable functionals with respect to the domain.
\end{abstract}

Key Words: Overdetermined problems, Domain derivative, Duality results, Domain functionals, p-Laplace equations, Generalized Monge-Ampére equations.

Mathematics Subject Classification: 35N25, 35A23, 35J96, 47J20, 52A40.

\section{Introduction}

Let $D$ be a bounded smooth domain in $\mathbb{R}^{N}$. A point $x \in D$ will be denoted with $x=\left(x^{1}, \cdots, x^{N}\right)$. We also denote $u_{i}=\frac{\partial u}{\partial x^{i}}, u_{i j}=\frac{\partial^{2} u}{\partial x^{2} \partial x^{j}}$, etc, the partial derivatives of $u$.

Let us recall the following well known overdetermined problem. Let $c$ be a constant. If there exists a solution $u$ to the Dirichlet problem

$$
\Delta u=1 \text { in } D, \quad u=0 \text { on } \partial D
$$

such that $u$ satisfies the additional condition

$$
|\nabla u|=c \text { on } \partial D
$$

*Behrouz.Emamizadeh@nottingham.edu.cn, School of Mathematical Sciences, The University of Nottingham Ningbo China

${ }^{\dagger}$ yichen.liu07@yahoo.com, Department of Mathematical Sciences, Xi'an Jiaotong-Liverpool University, Suzhou, China

${ }^{\ddagger}$ porru@unica.it, Department of Mathematics and Informatics, University of Cagliari, Italy 
then $D$ must be a ball. This result has been proved by J. Serrin [15] on 1971 using the moving plane method. At the same time, H. Weinberger [19] yields a different proof of the same result by using a Pohozaev identity and the maximum principle applied to a suitable P-function. The method of Weinberger requires less regularity of the boundary $\partial D$, but the method of Serrin can be easily applied to a large class of non-linear and fully non-linear operators. These two celebrated papers have inspired a great number of mathematicians, and the corresponding literature is nowadays very prominent. We refer to $[2,3,5,9$, $11,17]$ and references therein. For recent progress on this topic, we refer to the survey [10]. Among several ideas related to this overdetermined problem, we recall the following duality result [11].

Theorem 1.1 Let $u \in C^{2}(D) \cap C^{1}(\bar{D})$ be a solution to Problem (1). The following statements are equivalent:

(i) u satisfies condition (2).

(ii) The identity

$$
\int_{D} v d x=c \int_{\partial D} v d \sigma
$$

holds for all functions $v$ harmonic in $D$.

Motivated by this result, we shall prove duality theorems for overdetermined problems involving p-Laplace equations as well as generalized Monge-Ampére equations. In case of generalized Monge-Ampére equations, the overdetermined boundary condition is not the same as (2), but condition (27) below. In the linear case $(\kappa=1)$ condition (27) reduces to the familiar condition $|\nabla u|=c$ on $\partial D$. If $1<\kappa \leq N$, this condition involves $\nabla u$ as well as the second derivatives of $u$ throughout the Newton tensor $T_{\kappa-1}(u)$. Furthermore, we consider suitable functionals of the domain $D$ whose minimizers must satisfy the overdetermined boundary condition (2) for the p-Laplace problem, and condition (27) for the generalized Monge-Ampére problems. A crucial tool serving us shall be the domain derivative.

The paper is organized as follows. In Section 2 we introduce the notion of domain derivative. Some of our descriptions are formal, for a precise treatment of the domain derivative we refer to [16]. In particular, we find a sort of linearized equation of the p-Laplace equation $\Delta_{p} u=f(u)$ (see equation (10)), as well as a linearized equation of the generalized Monge-Ampére equation $S_{\kappa}(u)=$ $f(u)$ (see equation (19)). These linearized equations are crucial to get our duality results. Sections 3 and 4 contain our main results. Section 3 is made of two subsections. In Subsection 3.1 we prove a duality result for a p-Laplace boundary value problem (see Theorem 3.1). In Subsection 3.2 we prove a duality result for a boundary value problem corresponding to a generalized MongeAmpére equation (see Theorem 3.2). Also Section 4 is made of two subsections. In Section 4.1 we introduce a special functional associated with our p-Laplace equation in a domain $D$. We shall prove that the minimum of such functional with respect to $D$ under the condition $|D|=$ constant yields a condition for $\nabla u$ 
on $\partial D$ which is the same as used in Theorem 3.1 (i). In Section 4.2 we introduce a special functional associated with a generalized Monge-Ampére equation in a domain $D$. We shall prove that the minimum of such functional with respect to $D$ under the condition $|D|=$ constant yields a condition for $\nabla u$ on $\partial D$ which is the same as used in Theorem 3.2 (i).

\section{Domain derivative}

The theory of domain derivative is very useful in fields as shape optimization. From a mathematical point of view, it goes back to Hadamard [8] and Schiffer [14]. We recall shortly the definitions and refer to [16] for a careful treatment. If $\mathcal{L}(u)$ is a differential operator, we consider the Dirichlet problem:

$$
\mathcal{L}(u)=f(u) \text { in } D, \quad u=0 \text { on } \partial D,
$$

where $f$ is a smooth function such that problem (4) has a unique solution. Let $I$ be the identity map. For a smooth $\left(C^{2}\right.$ is enough) vector field $V: \mathbb{R}^{N} \rightarrow \mathbb{R}^{N}$, and $|t|$ small, define

$$
D^{t}=(I+t V)(D)
$$

Now, we consider the Dirichlet problem in $D^{t}$ :

$$
\mathcal{L}\left(u^{t}\right)=f\left(u^{t}\right) \text { in } D^{t}, \quad u^{t}=0 \text { on } \partial D^{t} .
$$

For $x \in D$ we define

$$
v(x)=\lim _{t \rightarrow 0} \frac{u^{t}(x)-u(x)}{t} .
$$

Clearly, since $D^{t}$ depends on the vector field $V$, also $v$ depends on $V$. By [16], $v$ satisfies the boundary condition

$$
v(x)=-\frac{\partial u}{\partial \nu}(V \cdot \nu) \text { on } \partial D
$$

where $\nu=\left(\nu^{1}, \cdots, \nu^{N}\right)$ is the unit exterior normal on $\partial D$.

To obtain the equation for $v$, we compute

$$
\lim _{t \rightarrow 0} \frac{1}{t}\left[\mathcal{L}\left(u^{t}\right)-\mathcal{L}(u)\right]=\lim _{t \rightarrow 0} \frac{1}{t}\left[f\left(u^{t}\right)-f(u)\right] .
$$

If $f$ is differentiable, we have

$$
f\left(u^{t}\right)-f(u)=f^{\prime}\left(u+\theta\left(u^{t}-u\right)\right)\left(u^{t}-u\right) . \quad 0<\theta<1 .
$$

Therefore,

$$
\lim _{t \rightarrow 0} \frac{1}{t}\left[f\left(u^{t}\right)-f(u)\right]=f^{\prime}(u) v
$$


The computation of the left hand side of (8) depends on the structure of the differential operator $\mathcal{L}$. If $\mathcal{L}(u)=\Delta u$ we find

$$
\lim _{t \rightarrow 0} \frac{1}{t}\left[\Delta u^{t}-\Delta u\right]=\Delta v
$$

Consider now the $p$-Laplacian $\mathcal{L}(u)=\operatorname{div}\left(|\nabla u|^{p-2} \nabla u\right)$. We have

$$
\left|\nabla u^{t}\right|^{p-2} \nabla u^{t}-|\nabla u|^{p-2} \nabla u=|\nabla u|^{p-2}\left(\nabla u^{t}-\nabla u\right)+\left(\left|\nabla u^{t}\right|^{p-2}-|\nabla u|^{p-2}\right) \nabla u^{t} .
$$

Therefore,

$\lim _{t \rightarrow 0} \frac{1}{t}\left(\left|\nabla u^{t}\right|^{p-2} \nabla u^{t}-|\nabla u|^{p-2} \nabla u\right)=|\nabla u|^{p-2} \nabla v+(p-2)|\nabla u|^{p-4}(\nabla u \cdot \nabla v) \nabla u$.

Hence, in this case, the equation corresponding to (8) for $v$ reads as

$$
\operatorname{div}\left(|\nabla u|^{p-2} \nabla v+(p-2)|\nabla u|^{p-4}(\nabla u \cdot \nabla v) \nabla u\right)=f^{\prime}(u) v .
$$

Now we recall the definition of generalized Monge-Ampére operators. Let $1 \leq \kappa \leq N$, and let $S_{\kappa}(u)$ be the $\kappa$-th elementary symmetric function of the eigenvalues of the Hessian matrix $H=D^{2} u=\left[u_{i j}\right]$ (that is, the sum of all principal minors of order $\kappa$ of $H$ ). Clearly, we have $S_{1}(u)=\Delta u$ (Laplace operator) and $S_{N}(u)=\operatorname{det}\left[D^{2} u\right]$ (Monge-Ampére operator). Given a positive smooth function $f(t)$, we consider the problem

$$
S_{\kappa}(u)=f(u) \text { in } D, \quad u=0 \text { on } \partial D .
$$

Suppose the domain $D \subset \mathbb{R}^{N}$ is bounded and smooth. In addition, for $\kappa$ fixed such that $2 \leq \kappa \leq N$, we assume the following property:

$$
\sigma_{\kappa-1} \geq \beta \quad \text { on } \partial D \text {, }
$$

where $\beta$ is a positive constant and $\sigma_{\kappa-1}$ is the $(\kappa-1)$-th elementary symmetric function of the principal curvatures of $\partial D$ with respect to its inner normal, see $[4,18]$. If we denote by $\tau_{1}, \tau_{2}, \cdots, \tau_{N-1}$ the principal curvatures of the surface $\partial D$ we have:

$$
\sigma_{1}=\sum_{1 \leq i \leq N-1} \tau_{i}, \quad \sigma_{2}=\sum_{1 \leq i_{1}<i_{2} \leq N-1} \tau_{i_{1}} \tau_{i_{2}}, \quad \sigma_{N-1}=\tau_{1} \tau_{2} \cdots \tau_{N-1} .
$$

Note that condition $\left(P_{N}\right)$ means that $\Omega$ is strictly convex. Moreover, if $\Omega$ enjoys property $\left(P_{\kappa}\right)$ then also $D^{t}=(I+t V)(D)$, for $|t|$ small, enjoys the same property (possibly with a smaller constant $\beta$ ). Finally, $f(t)$ is a positive smooth function such that problem (11) has a unique admissible solution. As usual, a solution is admissible if the operator $S_{\kappa}(u)$ is positive definite. In this situation, the 
solution $u$ is negative in $D$ and $\nu=\frac{\nabla u}{|\nabla u|}$ on the boundary $\partial D$. We refer to $[4,18]$ for a careful discussion of this problem.

It is convenient to define the matrix

$$
T_{\kappa-1}^{i j}(u)=\frac{\partial S_{\kappa}(u)}{\partial u_{i j}}, \quad i, j=1, \cdots, N .
$$

We put $T_{0}(u)=I$, the identity matrix. The matrix $T_{\kappa}(u)$ is known as the $\kappa$-th Newton tensor associated with $H$. We have [13]

$$
T_{\kappa}(u)=S_{\kappa}(u) I-T_{\kappa-1}(u) H, \quad \kappa=1, \cdots, N-1 .
$$

Since $H$ is symmetric, also $T_{\kappa}$ is symmetric. It has several nice properties. For example, we have

$$
\left(T_{\kappa}^{i j}(u)\right)_{i}=0, \quad j=1, \cdots, N
$$

where $\left(T_{\kappa}^{i j}(u)\right)_{i}=\frac{\partial T_{\kappa}^{i j}(u)}{\partial x^{i}}$, and here and in what follows, we use the summation convention over repeated indices from 1 to $N$. To prove (13), we recall the definition of the generalized Kronecker symbol

$$
\left(\begin{array}{llll}
i_{1} & i_{2} & \cdots & i_{q} \\
j_{1} & j_{2} & \cdots & j_{q}
\end{array}\right), \quad 2 \leq q \leq N
$$

where $i_{1}, \cdots, i_{q}$ are distinct integers between 1 and $N$, and also $j_{1}, \cdots, j_{q}$ are distinct integers between 1 and $N$. The value of the symbol is 1 (respectively $-1)$ if $\left(j_{1}, \cdots, j_{q}\right)$ is and even (respectively an odd) permutation of $\left(i_{1}, \cdots, i_{q}\right)$, and is 0 in all other cases. If $1 \leq \kappa \leq N-1$ we have (see [12])

$$
T_{\kappa}^{i j}(u)=\frac{1}{\kappa !}\left(\begin{array}{ccccc}
i_{1} & i_{2} & \cdots & i_{\kappa} & i \\
j_{1} & j_{2} & \cdots & j_{\kappa} & j
\end{array}\right) u_{i_{1} j_{1}} u_{i_{2} j_{2}} \cdots u_{i_{\kappa} j_{\kappa}} .
$$

We find

$$
\left(T_{\kappa}^{i j}(u)\right)_{i}=\frac{1}{\kappa !}\left(\begin{array}{ccccc}
i_{1} & i_{2} & \cdots & i_{\kappa} & i \\
j_{1} & j_{2} & \cdots & j_{\kappa} & j
\end{array}\right)\left(u_{i_{1} j_{1}} u_{i_{2} j_{2}} \cdots u_{i_{\kappa} j_{\kappa}}\right)_{i} .
$$

Simplifying we can write

$$
\left(T_{\kappa}^{i j}(u)\right)_{i}=\frac{1}{(\kappa-1) !}\left(\begin{array}{ccccc}
i_{1} & i_{2} & \cdots & i_{\kappa} & i \\
j_{1} & j_{2} & \cdots & j_{\kappa} & j
\end{array}\right) u_{i_{1} j_{1} i} u_{i_{2} j_{2}} \cdots u_{i_{\kappa} j_{\kappa}} .
$$

We note that $u_{i_{1} j_{1} i}$ is symmetric with respect to $i_{1} i$, while the Kronecker symbol is skew-symmetric with respect to those indices. Thus, the sum over $i_{1} i$ vanish, and (13) follows.

The proof in above can be extended to prove that, if also $v$ is a smooth function, we have

$$
\left(\begin{array}{ccccc}
i_{1} & i_{2} & \cdots & i_{\kappa} & i \\
j_{1} & j_{2} & \cdots & j_{\kappa} & j
\end{array}\right)\left(v_{i_{1} j_{1}} u_{i_{2} j_{2}} \cdots u_{i_{\kappa} j_{\kappa}}\right)_{i}=0, \quad j=1, \cdots, N .
$$


We refer to Proposition 2.1 of [12] for details.

Another very interesting property is the following (see $[12,13]$ )

$$
\frac{1}{\kappa} T_{\kappa-1}^{i j}(u) u_{i j}=S_{\kappa}(u), \quad \kappa=1, \cdots, N .
$$

We are now ready to find the equation for $v$ defined as in (6) with $\mathcal{L}(u)=$ $S_{\kappa}(u)$. Let $u^{t}$ be the (admissible) solution to problem (11) corresponding to $D^{t}$. Using (16) and (13) we have

$$
\begin{aligned}
& S_{\kappa}\left(u^{t}\right)-S_{\kappa}(u)=\frac{1}{\kappa}\left(T_{\kappa-1}^{i j}\left(u^{t}\right) u_{i j}^{t}-T_{\kappa-1}^{i j}(u) u_{i j}\right) \\
& =\frac{1}{\kappa}\left(T_{\kappa-1}^{i j}\left(u^{t}\right) u_{i}^{t}-T_{\kappa-1}^{i j}(u) u_{i}\right)_{j} \\
& =\frac{1}{\kappa}\left[\left(T_{\kappa-1}^{i j}(u)\left(u_{i}^{t}-u_{i}\right)\right)_{j}+\left(\left(T_{\kappa-1}^{i j}\left(u^{t}\right)-T_{\kappa-1}^{i j}(u)\right) u_{i}^{t}\right)_{j}\right] .
\end{aligned}
$$

We have

$$
\lim _{t \rightarrow 0} \frac{1}{t} T_{\kappa-1}^{i j}(u)\left(u_{i}^{t}-u_{i}\right)=T_{\kappa-1}^{i j}(u) v_{i} .
$$

Using (13) again we find

$$
\lim _{t \rightarrow 0} \frac{1}{t}\left(T_{\kappa-1}^{i j}(u)\left(u_{i}^{t}-u_{i}\right)\right)_{j}=T_{\kappa-1}^{i j}(u) v_{i j} .
$$

Moreover, using (14), we have

$$
\begin{aligned}
& \left(\left(T_{\kappa-1}^{i j}\left(u^{t}\right)-T_{\kappa-1}^{i j}(u)\right) u_{i}^{t}\right)_{j} \\
& =\frac{1}{\kappa !}\left(\begin{array}{ccccc}
i_{1} & i_{2} & \cdots & i_{\kappa-1} & i \\
j_{1} & j_{2} & \cdots & j_{\kappa-1} & j
\end{array}\right)\left(\left(u_{i_{1} j_{1}}^{t} u_{i_{2} j_{2}}^{t} \cdots u_{i_{\kappa-1} j_{\kappa-1}}^{t}-u_{i_{1} j_{1}} u_{i_{2} j_{2}} \cdots u_{i_{\kappa-1} j_{\kappa-1}}\right) u_{i}^{t}\right)_{j} .
\end{aligned}
$$

Hence,

$$
\begin{aligned}
& \lim _{t \rightarrow 0} \frac{1}{t}\left(\left(T_{\kappa-1}^{i j}\left(u^{t}\right)-T_{\kappa-1}^{i j}(u)\right) u_{i}^{t}\right)_{j} \\
& =\frac{1}{\kappa !}\left(\begin{array}{lllll}
i_{1} & i_{2} & \cdots & i_{\kappa-1} & i \\
j_{1} & j_{2} & \cdots & j_{\kappa-1} & j
\end{array}\right)\left(\left(v_{i_{1} j_{1}} u_{i_{2} j_{2}} \cdots u_{i_{\kappa-1} j_{\kappa-1}}+\cdots+u_{i_{1} j_{1}} u_{i_{2} j_{2}} \cdots v_{i_{\kappa-1} j_{\kappa-1}}\right) u_{i}\right)_{j} \\
& =\frac{\kappa-1}{\kappa !}\left(\begin{array}{ccccc}
i_{1} & i_{2} & \cdots & i_{\kappa-1} & i \\
j_{1} & j_{2} & \cdots & j_{\kappa-1} & j
\end{array}\right)\left(v_{i_{1} j_{1}} u_{i_{2} j_{2}} \cdots u_{i_{\kappa-1} j_{\kappa-1}} u_{i}\right)_{j} \\
& =\frac{\kappa-1}{\kappa !}\left(\begin{array}{ccccc}
i_{1} & i_{2} & \cdots & i_{\kappa-1} & i \\
j_{1} & j_{2} & \cdots & j_{\kappa-1} & j
\end{array}\right)\left(\left(v_{i_{1} j_{1}} u_{i_{2} j_{2}} \cdots u_{i_{\kappa-1} j_{\kappa-1}}\right)_{j} u_{i}+v_{i_{1} j_{1}} u_{i_{2} j_{2}} \cdots u_{i_{\kappa-1} j_{\kappa-1}} u_{i j}\right) .
\end{aligned}
$$


By using (15) and changing conveniently the indices we find

$$
\begin{gathered}
\lim _{t \rightarrow 0} \frac{1}{t}\left(\left(T_{\kappa-1}^{i j}\left(u^{t}\right)-T_{\kappa-1}^{i j}(u)\right) u_{i}^{t}\right)_{j}=\frac{\kappa-1}{\kappa !}\left(\begin{array}{lllll}
i_{1} & i_{2} & \cdots & i_{\kappa-1} & i \\
j_{1} & j_{2} & \cdots & j_{\kappa-1} & j
\end{array}\right) u_{i_{1} j_{1}} u_{i_{2} j_{2}} \cdots u_{i_{\kappa-1} j_{\kappa-1}} v_{i j} \\
=(\kappa-1) T_{\kappa-1}^{i j}(u) v_{i j},
\end{gathered}
$$

where (14) has been used once more. From (17), (18) and the latter result we find

$$
\lim _{t \rightarrow 0} \frac{S_{\kappa}\left(u^{t}\right)-S_{\kappa}(u)}{t}=\frac{1}{\kappa}\left(T_{\kappa-1}^{i j}(u) v_{i j}+(\kappa-1) T_{\kappa-1}^{i j}(u) v_{i j}\right)=T_{\kappa-1}^{i j}(u) v_{i j} .
$$

Hence, recalling (9), we find the equation for $v$ :

$$
T_{\kappa-1}^{i j}(u) v_{i j}=f^{\prime}(u) v .
$$

\section{Duality results}

In this section, we extend Theorem 1.1 to p-Laplace equations and to generalized Monge-Ampére equations.

\section{$3.1 \quad$ p-Laplace equations}

Let $D \subset \mathbb{R}^{N}$ be a bounded smooth domain, and let $f: \mathbb{R} \rightarrow \mathbb{R}$ be a $C^{1}$ positive function such that the problem

$$
\operatorname{div}\left(|\nabla u|^{p-2} \nabla u\right)=f(u) \text { in } D, \quad u=0 \text { on } \partial D,
$$

has a unique (negative) solution $u \in C^{1}(\bar{D}) \cap W^{1, p}(D)$. For example, one can take, for $\tau<0, f(\tau)=(-\tau)^{\alpha}, 0 \leq \alpha<p$, see [6]. We have

Theorem 3.1 Let $u$ be the solution to problem (20). Then the following statements are equivalent.

(i) There is a constant $c$ such that

$$
|\nabla u|=c \quad \text { on } \partial D
$$

(ii) There is a constant d such that

$$
\int_{D}\left(f(u)-\frac{1}{p-1} u f^{\prime}(u)\right) v d x=d \int_{\partial D} v d \sigma
$$

for all solutions $v$ to equation (10). 
Proof. Multiplying (10) by $-u$, integrating over $D$ and recalling that $u=0$ on $\partial D$ we find

$$
\begin{aligned}
& -\int_{D} f^{\prime}(u) v u d x=-\int_{D} \operatorname{div}\left(|\nabla u|^{p-2} \nabla v+(p-2)|\nabla u|^{p-4}(\nabla u \cdot \nabla v) \nabla u\right) u d x \\
& =\int_{D}\left(|\nabla u|^{p-2} \nabla v+(p-2)|\nabla u|^{p-4}(\nabla u \cdot \nabla v) \nabla u\right) \cdot \nabla u d x \\
& =(p-1) \int_{D}|\nabla u|^{p-2} \nabla u \cdot \nabla v d x \\
& =(p-1) \int_{\partial D}|\nabla u|^{p-2} \nabla u \cdot \nu v d \sigma-(p-1) \int_{D} \operatorname{div}\left(|\nabla u|^{p-2} \nabla u\right) v d x
\end{aligned}
$$

Since $\nu=\frac{\nabla u}{|\nabla u|}$ on $\partial D$, using equation (20), from the latter result we find

$$
\int_{D}\left(f(u)-\frac{1}{p-1} f^{\prime}(u) u\right) v d x=\int_{\partial D}|\nabla u|^{p-1} v d \sigma .
$$

If (i) holds, equation (23) yields (22) with $d=c^{p-1}$.

If (ii) holds, from equations (22) and (23) we find

$$
\int_{\partial D}|\nabla u|^{p-1} v d \sigma=d \int_{\partial D} v d \sigma .
$$

Using the boundary condition (7) we have

$$
v=-\frac{\partial u}{\partial \nu} V \cdot \nu=-|\nabla u| V \cdot \nu .
$$

Therefore, from (24) we find

$$
\int_{\partial D}\left(|\nabla u|^{p}-d|\nabla u|\right) V \cdot \nu d \sigma=0
$$

Since $V$ is arbitrary, we must have

$$
|\nabla u|\left(|\nabla u|^{p-1}-d\right)=0 \quad \text { on } \partial D \text {. }
$$

By Hopf's Lemma $|\nabla u|>0$, hence, $|\nabla u|=d^{\frac{1}{p-1}}$ on $\partial D$. The theorem is proved.

\subsection{Generalized Monge-Ampére equations}

Let $\kappa$ be an integer such that $1 \leq \kappa \leq N$. Let $D \subset \mathbb{R}^{N}$ be a bounded smooth domain satisfying property $\left(P_{\kappa}\right)$, and let $f: \mathbb{R} \rightarrow \mathbb{R}$ be a $C^{1}$ positive function such that the problem

$$
S_{\kappa}(u)=f(u) \text { in } D, \quad u=0 \text { on } \partial D,
$$

has a unique admissible solution $u \in C^{3}(D) \cap C^{1}(\bar{D})$. We have 
Theorem 3.2 Let $u$ be the admissible solution to problem (26). The following statements are equivalent.

(i) There is a constant $c$ such that

$$
T_{\kappa-1}^{i j}(u) u_{i} u_{j}=c^{2} \quad \text { on } \partial D
$$

(ii) There is a constant d such that

$$
\int_{D}\left(\kappa f(u)-u f^{\prime}(u)\right) v d x=d \int_{\partial D} \frac{v}{|\nabla u|} d \sigma
$$

hold for all solutions $v$ to equation (19).

Proof. Multiplying (19) by $-u$, integrating over $D$, using (13) and recalling that $u=0$ on $\partial D$ we find

$$
\begin{aligned}
& -\int_{D} u f^{\prime}(u) v d x=-\int_{D} u T_{\kappa-1}^{i j}(u) v_{i j} d x \\
& =-\int_{D} u\left(T_{\kappa-1}^{i j}(u) v_{i}\right)_{j} d x=\int_{D} u_{j} T_{\kappa-1}^{i j}(u) v_{i} d x .
\end{aligned}
$$

Integrating by parts and using (13) again we find

$$
\int_{D} u_{j} T_{\kappa-1}^{i j}(u) v_{i} d x=\int_{\partial D} u_{j} T_{\kappa-1}^{i j}(u) v \nu^{i} d \sigma-\int_{D} u_{i j} T_{\kappa-1}^{i j}(u) v d x .
$$

Since $T_{\kappa-1}^{i j}(u) u_{i j}=\kappa f(u)$ in $D$ and $\nu^{i}|\nabla u|=u_{i}$ on $\partial D$, from the latter equation we find

$$
\int_{D} u_{j} T_{\kappa-1}^{i j}(u) v_{i} d x=\int_{\partial D} T_{\kappa-1}^{i j}(u) u_{i} u_{j} \frac{v}{|\nabla u|} d \sigma-\int_{D} \kappa f(u) v d x
$$

From (29) and (30) it follows that

$$
\int_{D}\left(\kappa f(u)-u f^{\prime}(u)\right) v d x=\int_{\partial D} T_{\kappa-1}^{i j}(u) u_{i} u_{j} \frac{v}{|\nabla u|} d \sigma .
$$

If (i) holds, equation (31) yields (28) with $d=c^{2}$.

If (ii) holds, from equations (28) and (31) we find

$$
\int_{\partial D}\left[T_{\kappa-1}^{i j}(u) u_{i} u_{j}-d\right] \frac{v}{|\nabla u|} d \sigma=0 .
$$

Finally, using the boundary condition (7) we get

$$
\int_{\partial D}\left[T_{\kappa-1}^{i j}(u) u_{i} u_{j}-d\right] V \cdot \nu d \sigma=0 .
$$

Since $V$ is arbitrary, (27) follows with $c^{2}=d$. The theorem is proved.

Let us recall a result from [1]. 
Theorem 3.3 Let $D$ be a bounded convex domain in the plane and let $c$ be a constant. If there exists a convex solution $u$ to the Dirichlet problem

$$
S_{2}(u)=u_{11} u_{22}-u_{12}^{2}=1 \text { in } D, \quad u=0 \text { on } \partial D
$$

such that $u$ satisfies the additional condition

$$
u_{22} u_{1}^{2}+u_{11} u_{2}^{2}-2 u_{12} u_{1} u_{2}=c^{2} \text { on } \partial D,
$$

then $D$ must be an ellipse.

Proof. See Theorem 2.4 of [1].

Corollary 3.4 Let $D$ be a bounded convex domain in the plane and let $c$ be a constant. If there exists a convex solution $u$ to problem (33) such that the integral equations

$$
2 \int_{D} v d x=c^{2} \int_{\partial D} \frac{v}{|\nabla u|} d s
$$

hold for all solutions $v$ to the equation

$$
u_{22} v_{11}+u_{11} v_{22}-2 u_{12} v_{12}=0 \text { in } D,
$$

then, $D$ is an ellipse.

Proof. Since $T_{1}^{11}(u)=u_{22}, T_{1}^{12}(u)=T_{1}^{21}(u)=-u_{12}$ and $T_{1}^{22}(u)=u_{11}$, equation (36) can be written as $T_{1}^{i j}(u) v_{i j}=0$, and condition (34) can be written as $T_{1}^{i j} u_{i} u_{j}=c^{2}$. Hence, the corollary follows from Theorem 3.2 and Theorem 3.3.

\section{Minimization of functionals}

In this section we present a motivation of the overdetermined conditions (2) and (27).

\section{$4.1 \quad \mathrm{p}$-Laplace equations}

Let $D \subset \mathbb{R}^{N}$ be a bounded smooth domain, and recall the problem (20) below

$$
\operatorname{div}\left(|\nabla u|^{p-2} \nabla u\right)=f(u) \text { in } D, \quad u=0 \text { on } \partial D,
$$

where $f$ is a positive function such that problem (37) has a unique (negative) solution. Given $D$ and the corresponding solution $u$ to problem (37), we consider the functional

$$
J(D)=\int_{D}\left(|\nabla u|^{p}+p \int_{0}^{u} f(\tau) d \tau\right) d x
$$


Theorem 4.1 Let $J(D)$ be defined as in (38). If $\hat{D}$ is a minimum of $J(D)$ among all domains $D$ having the same measure as $\hat{D}$, then $|\nabla u|$ is constant on $\partial \hat{D}$.

Proof. To prove the theorem, we use the notion of domain derivative. Recall that $I$ is the identity map. Let $V: \mathbb{R}^{N} \rightarrow \mathbb{R}^{N}$ be a smooth vector field, and let $D^{t}=(I+t V) \hat{D}$ be a deformation of $\hat{D}$. By the well known Lagrange principle, $\hat{D}$ is a stationary point of the functional

$$
I(D) \equiv J(D)+\lambda(K(D)-\mu), \quad K(D)=|D|, \quad \mu=|\hat{D}|,
$$

where $\lambda$ is a real parameter. Since $\hat{D}$ is a stationary point of $I(D)$, we must have $d I(\hat{D}, V)=0$ for every vector field $V$. Clearly,

$$
d I(\hat{D}, V) \equiv d J(\hat{D}, V)+\lambda d K(\hat{D}, V) .
$$

We compute first $d J(\hat{D}, V)$. Let $u$ be the solution of problem (37) with $D=\hat{D}$, and let $u^{t}$ be the solution of problem (37) corresponding to $D^{t}$. We have

$$
\begin{aligned}
& d J(\hat{D}, V)=\lim _{t \rightarrow 0} \frac{J\left(D^{t}\right)-J(\hat{D})}{t} \\
& =\lim _{t \rightarrow 0} \frac{1}{t}\left[\int_{D^{t}}\left(\left|\nabla u^{t}\right|^{p}+p \int_{0}^{u^{t}} f(\tau) d \tau\right) d x-\int_{\hat{D}}\left(|\nabla u|^{p}+p \int_{0}^{u} f(\tau) d \tau\right) d x\right] \\
& =\lim _{t \rightarrow 0} \frac{1}{t}\left[\int_{D^{t}}\left(\left|\nabla u^{t}\right|^{p}+p \int_{0}^{u^{t}} f(\tau) d \tau\right) d x-\int_{\hat{D}}\left(\left|\nabla u^{t}\right|^{p}+p \int_{0}^{u^{t}} f(\tau) d \tau\right) d x\right] \\
& +\lim _{t \rightarrow 0} \frac{1}{t}\left[\int_{\hat{D}}\left(\left|\nabla u^{t}\right|^{p}+p \int_{0}^{u^{t}} f(\tau) d \tau\right) d x-\int_{\hat{D}}\left(|\nabla u|^{p}+p \int_{0}^{u} f(\tau) d \tau\right) d x\right] .
\end{aligned}
$$

Since $u=0$ on $\partial \hat{D}$ we find

$$
\begin{aligned}
& \lim _{t \rightarrow 0} \frac{1}{t}\left[\int_{D^{t}}\left(\left|\nabla u^{t}\right|^{p}+p \int_{0}^{u^{t}} f(\tau) d \tau\right) d x-\int_{\hat{D}}\left(\left|\nabla u^{t}\right|^{p}+p \int_{0}^{u^{t}} f(\tau) d \tau\right) d x\right] \\
& =\int_{\partial \hat{D}}\left(|\nabla u|^{p}+p \int_{0}^{u} f(\tau) d \tau\right) V \cdot \nu d \sigma=\int_{\partial \hat{D}}|\nabla u|^{p} V \cdot \nu d \sigma .
\end{aligned}
$$

Therefore, we find

$$
\begin{aligned}
& d J(\hat{D}, V)=\int_{\partial \hat{D}}|\nabla u|^{p} V \cdot \nu d \sigma \\
& +\lim _{t \rightarrow 0} \int_{\hat{D}} \frac{\left|\nabla u^{t}\right|^{p}-|\nabla u|^{p}}{t} d x+p \lim _{t \rightarrow 0} \int_{\hat{D}} \frac{\int_{u}^{u^{t}} f(\tau) d \tau}{t} d x \\
& =\int_{\partial \hat{D}}|\nabla u|^{p} V \cdot \nu d \sigma+p\left(\int_{\hat{D}}|\nabla u|^{p-2} \nabla u \cdot \nabla v d x+\int_{\hat{D}} f(u) v d x\right),
\end{aligned}
$$

where $v$ is defined as

$$
v(x)=\lim _{t \rightarrow 0} \frac{u^{t}(x)-u(x)}{t} .
$$


Integrating the equation

$$
-v \operatorname{div}\left(|\nabla u|^{p-2} \nabla u\right)=-f(u) v
$$

over $\hat{D}$ we find

$$
-\int_{\partial \hat{D}} v|\nabla u|^{p-2} \nabla u \cdot \nu d \sigma+\int_{\hat{D}}|\nabla u|^{p-2} \nabla u \cdot \nabla v d x=-\int_{\hat{D}} f(u) v d x .
$$

Recalling that $\nabla u=|\nabla u| \nu$ on $\partial D$ and using the boundary condition (7), from the latter equation we find

$$
\int_{\hat{D}}|\nabla u|^{p-2} \nabla u \cdot \nabla v d x+\int_{\hat{D}} f(u) v d x=-\int_{\partial \hat{D}}|\nabla u|^{p} V \cdot \nu d \sigma .
$$

By (40) and the latter equation we find

$d J(\hat{D}, V)=\int_{\partial \hat{D}}|\nabla u|^{p} V \cdot \nu d \sigma-p \int_{\partial \hat{D}}|\nabla u|^{p} V \cdot \nu d \sigma=-(p-1) \int_{\partial \hat{D}}|\nabla u|^{p} V \cdot \nu d \sigma$.

On the other hand (see [16] page 652 formula (12) with $C(u)=1$ ), we have

$$
d K(\hat{D}, V)=\int_{\partial \hat{D}} V \cdot \nu d \sigma .
$$

Insertion of (41) and (42) into (39) yields

$$
\begin{aligned}
d I(\hat{D}, V) & =-(p-1) \int_{\partial \hat{D}}|\nabla u|^{p} V \cdot \nu d \sigma+\lambda \int_{\partial \hat{D}} V \cdot \nu d \sigma \\
& =\int_{\partial \hat{D}}\left(-(p-1)|\nabla u|^{p}+\lambda\right) V \cdot \nu d \sigma .
\end{aligned}
$$

Since $d I(\hat{D}, V)=0$ for every vector field $V$, it follows that $|\nabla u|^{p}=\frac{\lambda}{p-1}$. Therefore, $|\nabla u|$ is a constant on $\partial \hat{D}$, and the theorem is proved.

\subsection{Generalized Monge-Ampére equations}

Now we prove a similar result for generalized Monge-Ampére equations. Assume the domain $D$ bounded, smooth and having the property $\left(P_{\kappa}\right)$. Let $u$ be an admissible solution to the problem

$$
S_{\kappa}(u)=f(u) \quad \text { in } D, u=0 \text { on } \partial D .
$$

Here $1 \leq \kappa \leq N$ and $f(t)>0$. Consider the functional

$$
E(D)=\int_{D}\left(T_{\kappa-1}^{i j}(u) u_{i} u_{j}+\left(\kappa+\kappa^{2}\right) \int_{0}^{u} f(\tau) d \tau\right) d x,
$$

where $u$ is an admissible solution to problem (43). 
Theorem 4.2 Let $E(D)$ be defined as in (44). If $\hat{D}$ is a minimum of $E(D)$ among all domains $D$ having the property $\left(P_{\kappa}\right)$ and having the same measure as $\hat{D}$, then we have

$$
T_{\kappa-1}^{i j}(u) u_{i} u_{j}=\text { constant } \quad \text { on } \partial \hat{D} .
$$

Proof. Let us find a different formulation for $E(D)$. If we multiply (43) by $u$ and use (16) we have

$$
T_{\kappa-1}^{i j}(u) u_{i j} u=\kappa f(u) u .
$$

Integration over $D$ yields

$$
-\int_{D} T_{\kappa-1}^{i j}(u) u_{i} u_{j} d x=\kappa \int_{D} f(u) u d x .
$$

Hence, the functional defined by (44) can be rewritten as

$$
E(D)=\int_{D}\left(-\kappa f(u) u+\left(\kappa+\kappa^{2}\right) \int_{0}^{u} f(\tau) d \tau\right) d x .
$$

From now on, we shall use this formula for $E(D)$.

By the well known Lagrange principle, $\hat{D}$ is a stationary point of the functional

$$
I(D) \equiv E(D)+\lambda(K(D)-\mu), \quad K(D)=|D|, \quad \mu=|\hat{D}|,
$$

where $\lambda$ is a real parameter. For a smooth vector field $V$, let $D^{t}=(I+t V) \hat{D}$ be a deformation of $\hat{D}$. We must have $d I(\hat{D}, V)=0$ for every vector field $V$. Clearly,

$$
d I(\hat{D}, V) \equiv d E(\hat{D}, V)+\lambda d K(\hat{D}, V) .
$$

If $u^{t}$ is the solution to problem (43) corresponding to $D^{t}$, we compute

$$
\begin{aligned}
& d E(\hat{D}, V)=\int_{\partial \hat{D}}\left(-\kappa f(u) u+\left(\kappa+\kappa^{2}\right) \int_{0}^{u} f(\tau) d \tau\right) V \cdot \nu d \sigma \\
& +\lim _{t \rightarrow 0} \frac{1}{t} \int_{\hat{D}}\left(-\kappa\left(f\left(u^{t}\right) u^{t}-f(u) u\right)+\left(\kappa+\kappa^{2}\right) \int_{u}^{u^{t}} f(\tau) d \tau\right) d x .
\end{aligned}
$$

Since $u=0$ on $\partial \hat{D}$, the first integral vanishes. Hence,

$$
\begin{aligned}
d E(\hat{D}, V) & =\int_{\hat{D}}\left(-\kappa\left(f^{\prime}(u) v u+f(u) v\right)+\left(\kappa+\kappa^{2}\right) f(u) v\right) d x \\
& =\int_{\hat{D}}\left(-\kappa f^{\prime}(u) v u+\kappa^{2} f(u) v\right) d x .
\end{aligned}
$$

As usual, the function $v$ is defined as

$$
v(x)=\lim _{t \rightarrow 0} \frac{u^{t}(x)-u(x)}{t} .
$$


Now we multiply equation (19) by $-u$ and integrate over $\hat{D}$. We find

$$
\begin{aligned}
& -\int_{\hat{D}} f^{\prime}(u) v u d x=-\int_{\hat{D}} T_{\kappa-1}^{i j}(u) v_{i j} u d x=\int_{\hat{D}} T_{\kappa-1}^{i j}(u) u_{i} v_{j} d x \\
& =\int_{\partial \hat{D}} T_{\kappa-1}^{i j}(u) u_{i} \nu^{j} v d \sigma-\int_{\hat{D}} T_{\kappa-1}^{i j}(u) u_{i j} v d x .
\end{aligned}
$$

Since $\nu^{j}|\nabla u|=u_{j}$ and $v=-|\nabla u| V \cdot \nu$ on $\partial \hat{D}$, and $T_{\kappa-1}^{i j}(u) u_{i j}=\kappa f(u)$ in $\hat{D}$, from the latter equation we find

$$
-\int_{\hat{D}} \kappa f^{\prime}(u) v u d x=-\kappa \int_{\partial \hat{D}} T_{\kappa-1}^{i j}(u) u_{i} u_{j} V \cdot \nu d \sigma-\int_{\hat{D}} \kappa^{2} f(u) v d x .
$$

In view of the latter result, from (46) we get

$$
d E(\hat{D}, V)=-\kappa \int_{\partial \hat{D}} T_{\kappa-1}^{i j}(u) u_{i} u_{j} V \cdot \nu d \sigma
$$

Insertion of (47) and (42) into (45) yields

$$
d I(\hat{D}, V)=\int_{\partial \hat{D}}\left(-\kappa T_{\kappa-1}^{i j}(u) u_{i} u_{j}+\lambda\right) V \cdot \nu d \sigma .
$$

Since $d I(\hat{D}, V)=0$ for every vector field $V$, it follows that $T_{\kappa-1}^{i j}(u) u_{i} u_{j}=\frac{\lambda}{\kappa}$. Therefore, $T_{\kappa-1}^{i j}(u) u_{i} u_{j}$ is a constant on $\partial \hat{D}$, and the theorem is proved.

Corollary 4.3 Let $D$ be a convex planar domain, let $u$ be a convex solution to problem (43) with $N=\kappa=2$ and $f=1$. If $E(D)$ is the corresponding functional defined as in (44), and if $\hat{D}$ is a minimum of $E(D)$ among all convex domains $D$ having the same measure as $\hat{D}$, then $D$ is an ellipse.

Proof. It follows from Theorems 4.2 and 3.3 .

Acknowledgements. Y. Liu is partly supported by the Natural Science Foundation of Jiangsu Province [grant number SBK2020041299].

\section{References}

[1] C. Anedda, G. Porru, Problems on the Monge-Ampére equation in the plane, Contemporary Mathematics, Volume 400 (2006), 1-9.

[2] A. Bennett, Symmetry in an ovedetermined fourth order elliptic boundary value problem, SIAM J. Math. Anal., 17 (1986), 1354-1358.

[3] B. Brandolini, C. Nitsch, P. Salani, C. Trombetti, Serrin type overdetermined problems: An alternative proof, Archive for Rational Mechanics and Analysis., 190 (2008), 267-280. 
[4] L. Caffarelli, L. Nirenberg, J. Spruck, Dirichlet problem for nonlinear second order elliptic equations III. Functions of the eigenvalues of the Hessian. Acta Math., 155 (1985), 261-301.

[5] M. Choulli, A. Henrot, Use of the domain derivative to prove symmetry results in partial differential equations, Math. Nachr., 192 (1998), 91-103.

[6] F. Cuccu, G. Porru, S. Sakaguchi, Optimization problems on general classes of rearrangements, Nonlinear Analysis, 74 (2011), 5554-5565.

[7] N. Garofalo, J.L. Lewis, A symmetry result related to some overdetermined boundary value problems, American J. of Math., 111 (1989), no. 1, 9-33.

[8] J. Hadamard, Memoires sur le probleme d'analyse relatif a l'equilibre des plaque elastique encastrees, Mem. Savants Etrangers, 33 (1908).

[9] R. Magnanini, Alexandrov, Serrin, Weinberger, Reilly: symmetry and stability by integral identities, Bruno Pini Mathematical Seminar, (2017), $121-141$.

[10] C. Nitsch, C. Trombetti, The classical overdetermined Serrin problem, Complex Var. Elliptic Equ., 63 (2018), no. 7-8, 1107-1122.

[11] L.E. Payne, P.W. Schaefer, Duality theorems in some overdetermined problems, Math. Methods in the Appl. Sciences, 11 (1989), 805-819.

[12] R.C. Reilly, On the Hessian of a function and the curvatures of its gragh, Michigan Math. J., 20 (1973), 373-383.

[13] R.C. Reilly, Variational properties of functions of the mean curvatures for hypersurfaces in space forms, J. Differential Geom., 8 (1973), 465-477.

[14] M. Schiffer, Variation of domain functionals, Bull. Amer. Math. Soc., 60 (1954), 303-328.

[15] J. Serrin, A symmetry problem in potential theory, Arch. Rational Mech. Anal., 43 (1971), 304-318.

[16] J. Simon, Differentiation with respect to the domain in boundary value problems. Numer. Funct. Anal. and Optimization, (1980), 649-687.

[17] A. Wagner, Pohozaev's Identity from a Variational Viewpoint, J. Math. Anal. Appl., 266 (2002), 149-159.

[18] X. J. Wang, The k-Hessian equation, Geometric Analysis and PDEs, Lecture Notes in Mathematics 1977, Springer-Verlag, Dordrecht (2009), 177252.

[19] H.F. Weinberger, Remark on the preceding paper of Serrin, Arch. Rational Mech. Anal., 43 (1971), 319-320. 